Corrigendum

\title{
Corrigendum to "Effects of a Mindfulness Meditation Course on Learning and Cognitive Performance among University Students in Taiwan"
}

\author{
Hoi-Ching Ho, ${ }^{1}$ Malcolm Koo, ${ }^{2,3}$ Tsung-Huang Tsai, ${ }^{4}$ and Chiu-Yuan Chen ${ }^{1,5}$ \\ ${ }^{1}$ Department of Natural Biotechnology, Nanhua University, Dalin, Chiayi 62249, Taiwan \\ ${ }^{2}$ Department of Medical Research, Dalin Tzu Chi Hospital, Buddhist Tzu Chi Medical Foundation, Dalin, Chiayi 62247, Taiwan \\ ${ }^{3}$ Dalla Lana School of Public Health, University of Toronto, Toronto, ON, Canada M5T 3M7 \\ ${ }^{4}$ Department of Psychiatry, Dalin Tzu Chi Hospital, Buddhist Tzu Chi Medical Foundation, Dalin, Chiayi 62247, Taiwan \\ ${ }^{5}$ Research and Extension Center of Natural Healing Sciences, Nanhua University, Dalin, Chiayi 62249, Taiwan \\ Correspondence should be addressed to Chiu-Yuan Chen; chiuyuan@mail.nhu.edu.tw \\ Received 20 December 2015; Accepted 31 December 2015 \\ Copyright (c) 2016 Hoi-Ching Ho et al. This is an open access article distributed under the Creative Commons Attribution License, \\ which permits unrestricted use, distribution, and reproduction in any medium, provided the original work is properly cited.
}

The first author's name should be Hoi-Ching Ho in the paper titled "Effects of a Mindfulness Meditation Course on Learning and Cognitive Performance among University Students in Taiwan" [1].

\section{References}

[1] H.-H. Ching, M. Koo, T.-H. Tsai, and C.-Y. Chen, "Effects of a mindfulness meditation course on learning and cognitive performance among university students in Taiwan," EvidenceBased Complementary and Alternative Medicine, vol. 2015, Article ID 254358, 7 pages, 2015. 


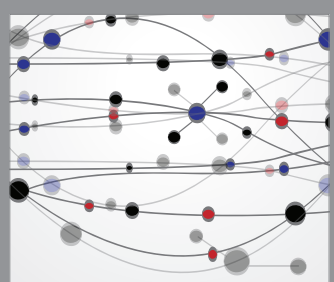

The Scientific World Journal
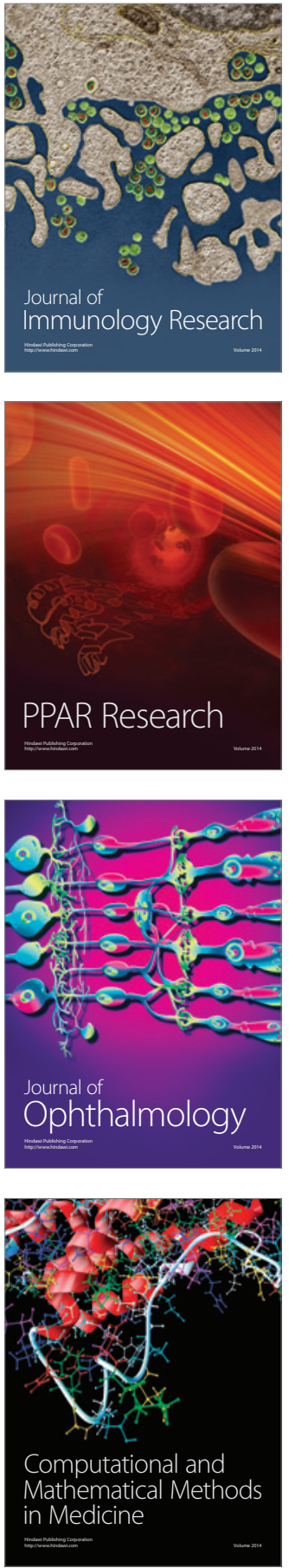

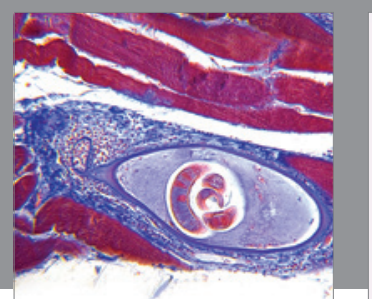

Gastroenterology Research and Practice

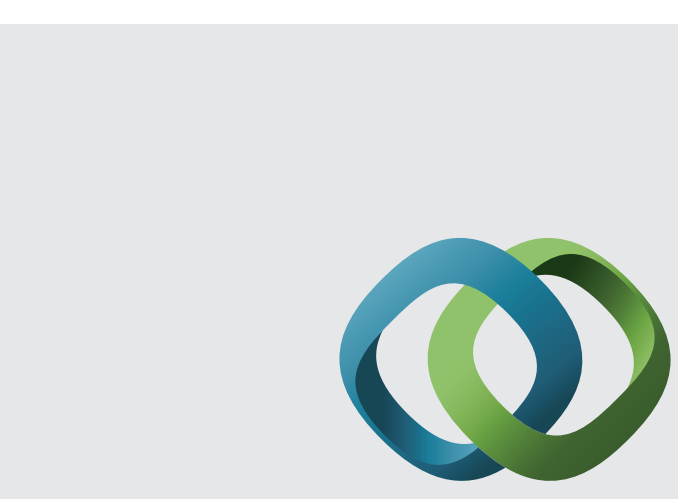

\section{Hindawi}

Submit your manuscripts at

http://www.hindawi.com
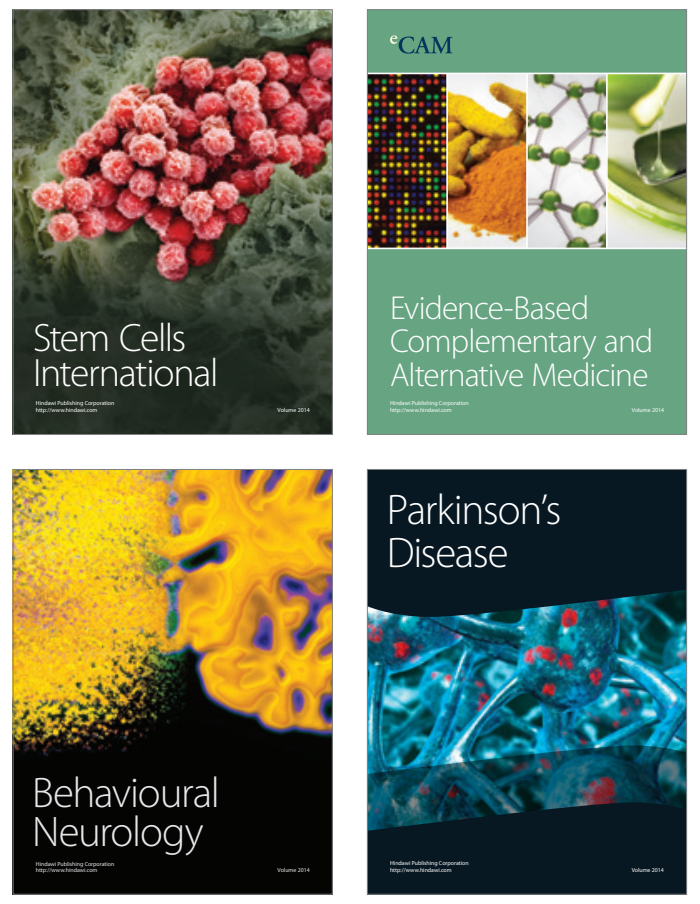
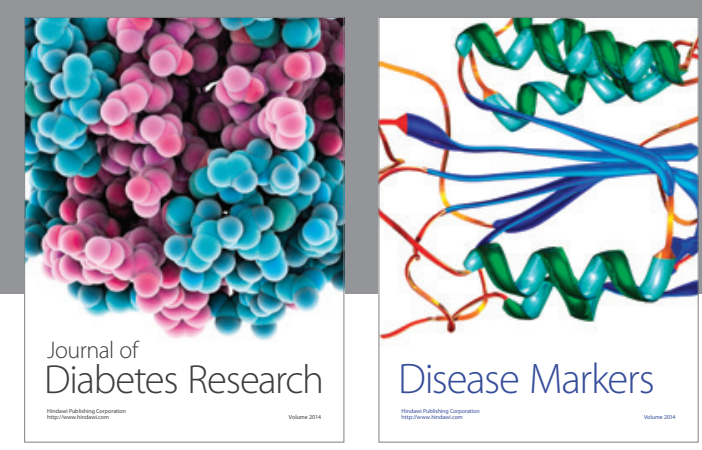

Disease Markers
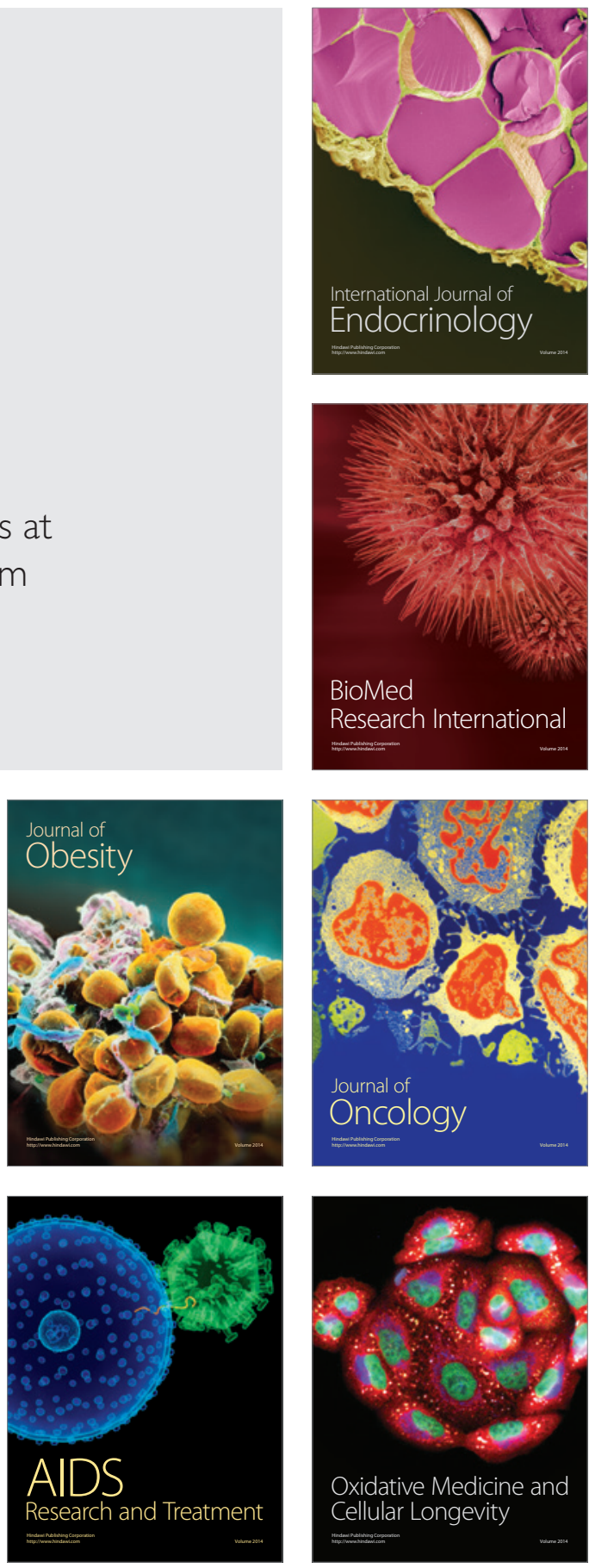